Original Article

\title{
Delphi consensus on the current clinical and therapeutic knowledge on Anderson-Fabry disease
}

\author{
Daniela Concolino ${ }^{\mathrm{a}}$, Emilia Degennaro ${ }^{\mathrm{b}}$, Rossella Parini ${ }^{\mathrm{c}, *}$, on behalf of the Fabry Delphi working group ${ }^{1}$ \\ a Department of Paediatrics, University Magna Graecia of Catanzaro, Catanzaro, Italy \\ b Graduate School in Teaching, University Roma Tre, Rome, Italy \\ c Rare Metabolic Diseases Unit, Paediatric Clinic, San Gerardo Hospital, University Milano Bicocca, Monza, Italy
}

\section{A R T I C L E I N F O}

Article history:

Received 28 May 2014

Received in revised form 21 July 2014

Accepted 24 July 2014

Available online 12 August 2014

\section{Keywords:}

Fabry disease

Diagnosis

Treatment

Management

\begin{abstract}
A B S T R A C T
Background: Management of Anderson-Fabry disease (AFD) is contentious, particularly regarding enzyme replacement therapy (ERT). We report results of a Delphi consensus panel on AFD management.

Methods: A survey to gauge consensus among AFD experts was distributed online and responses were analysed. Statements on: 1) diagnosis; 2) when starting ERT; 3) management of ERT infusion and adverse reactions; and 4) follow-up/monitoring response to therapy and progression of disease were included. Responses without consensus were discussed with an enlarged panel and modified to reach consensus.

Results: 15 experts responded to the survey. After plenary discussion among the enlarged panel, consensus was reached on most statements. Key points were the use of a target organ biopsy to show Gb3 deposits in symptomatic women with negative molecular analysis, the need for ERT in symptomatic women and in all patients with persistent signs and symptoms \pm organ damage. It was agreed to assess vital signs before ERT administration and use a $0.2 \mu \mathrm{L}$ filter on infusion to reduce the risk of adverse reactions, that serum should be drawn prior to the first infusion for anti-agalsidase antibody analysis to have a baseline value if a subsequent adverse reaction appears, and that pre-medication is required in those with prior infusion reactions. Holter ECG monitoring, cardiac and brain MRI, renal parameters, and abdominal ultrasound were considered important for the assessment of disease progression and response at ERT.

Conclusions: This consensus supplies guidance to healthcare providers on best practice in the management of patients with AFD and indicates a need for more guidance.
\end{abstract}

(c) 2014 European Federation of Internal Medicine. Published by Elsevier B.V. All rights reserved.

\section{Introduction}

Despite the growing interest and an increasing amount of published literature and clinical data on Anderson-Fabry disease (AFD) [1] there are still uncertainties in terms of disease management [2]. AFD is an $\mathrm{X}$-linked progressive multi-organ, metabolic lysosomal storage disorder caused by deficiency in the lysosomal enzyme alpha-galactosidase A leading to accumulation of glycosphingolipids (mainly globotriaosylceramide [Gb3]) in vascular endothelial and smooth muscle cells, cardiac myocytes,

\footnotetext{
* Corresponding autor at: UOS Malattie Metaboliche Rare, Clinica Pediatrica, Ospedale San Gerardo, Via Pergolesi 33, Monza, Italy. Tel.: + 39 2333286; fax: + 392334364

E-mail addresses: dconcolino@unicz.it (D. Concolino),degennaro@tin.it

(E. Degennaro), rossella.parini@unimib.it (R. Parini).

1 Delphi working group list of collaborators (alphabetical order): Daniela Antuzzi Bruno Bembi; Andrea Benso; Gianni Carraro; Cristina Chimenti; Loredana Colla; Maria Teresa Cuonzo; Goffredo Del Rosso; Marina Diomedi; Claudio Feliciani; Sandro Feriozzi Anna Ficcadenti; Andrea Frustaci; Maria Gnarra; Margherita Maccarone; Michelangelo Mancuso; Andrea Matucci; Renzo Mignani; Beatrice Musumeci; Patrizia Nencini; Stefania Piga; Antonio Pisani; Federica Re; Alessandro Salviati; Marco Spada; Alessandra Vultaggio; Elisabetta Zachara; Maria Luisa Zedde; Pier Giorgio Zoli.
}

dorsal root ganglion neurons, neurons of the autonomic nervous system, brain, all types of kidney cells, gastrointestinal tract, and elsewhere, leading to multisystemic disease. Clinical manifestations include acroparaesthesia, pain crises, hyper/hypo/anhydrosis, angiokeratomas, gastrointestinal symptoms, corneal dystrophy, hearing impairment, heat and cold sensitivity, and renal, cardiac and cerebrovascular disease. Due to the X-linked inheritance, the disease primarily affects males, but females can be affected as well. Although the biological basis is not fully understood, the random X-chromosomal inactivation might have a role. However, the onset and rate of disease progression are more variable in female carriers than in hemizygous males, with a tendency to occur later in life [3]. Even if clinical manifestations arise in childhood in either sex, due to the rarity of disease and to the heterogeneous phenotypic expression, diagnosis is often challenging and is reached in adult age for many patients. The most frequent symptoms reported by paediatric patients with AFD are recurrent neuropathic pain in hands and feet (acroparaesthesia) and gastrointestinal problems (mainly abdominal pain and diarrhoea) [4]. With age, progressive damage to vital organ systems develops in both genders, leading to organ failure, end-stage renal disease and life-threatening cardiovascular or cerebrovascular 
complications limiting life-expectancy [5]. Enzymatic activity and molecular analysis of the gene confirm the clinical diagnosis. Enzyme activity in leukocytes is always very low in affected males but may be normal or borderline normal in symptomatic affected females whose diagnosis needs to be confirmed by the genetic analysis. Current treatments consist of enzyme replacement therapy (ERT) and palliative treatments [6-13]. Two agalsidase preparations, agalsidase alfa and agalsidase beta, are available for ERT.

In addition, goals of treatment are not clearly defined, due in part to the heterogeneity of the disease [1]. Moreover, AFD patients can be treated in a range of settings from specialist reference centres to nonspecialist therapeutic units, therefore a clear guidance on AFD is welcome.

\subsection{Objectives}

The objective of this paper is to report the results generated by a Delphi consensus panel on some unanswered questions related to clinical and therapeutic aspects of AFD.

\section{Materials and methods}

\subsection{The Delphi process}

The rationale for using the Delphi method to achieve consensus has been previously published [14]: the Delphi method is a way of collecting opinions from experts - "a Delphi panel" - and is widely applied in various fields, including healthcare, to obtain consensus or to provide recommendations on a well-defined and specified topic. Although often referred to as a 'panel', the experts provide their opinions freely, individually and anonymously and this method provides a quick and economic way to contact a large group of experts.

\subsection{Phase 1 - pre-meeting}

A survey (see Appendix A), designed to gauge the level of consensus among a group of expert health professionals from well-known centres of excellence in the diagnosis and management of AFD, was created by two experts (DC and RP) and distributed online to participating clinicians; their responses were then collected anonymously and analysed prior to the enlarged meeting.

Participants voted using a 5-point scale to indicate their level of agreement on each statement $(1=$ absolutely disagree, $2=$ disagree, 3 = agree, $4=$ more than agree, $5=$ absolutely agree). Consensus was reached when the sum of items $1+2$ or $3+4+5$ exceeded $66 \%$.

The coordinators evaluated the responses and gathered those for which there was no consensus.

\subsection{Phase II - Delphi panel}

Experts from a range of fields participated in the face-to-face Delphi panel discussions, ensuring a multidisciplinary approach, and allowing opinions and views from different perspectives to be expressed. Participants' specialty, the number of follow-up patients they have with AFD, and the proportion of infused patients, were recorded.

Statements were divided into 4 main areas: 1) diagnosis; 2) starting ERT; 3) management of ERT infusion and adverse reactions; and 4) tests to evaluate the response to therapy and disease progression (follow-up of patients with AFD). Statements without consensus were selected for discussion in the Delphi plenary session. After discussion, and modification of the statements if required, participants reflected on the comments raised in the discussion and voted again using the same 5-point scale.

\section{Results}

\subsection{Participants}

Fifteen experts responded to the survey. They were from the different specialties involved in the management of patients with AFD: nephrology, endocrinology, cardiology, neurology, immunology, paediatrics and dermatology. Their expertise in dealing with AFD was based on experience gained on diagnosing and treating more than three patients.

\subsection{Overall consensus}

Four statements for which consensus was not achieved in the survey were selected for discussion in the Delphi plenary session of 50 experts. After discussion of the statements, second votes were taken and, after the second vote, consensus was reached on one statement; therefore, consensus ( $>66 \%$ either positive or negative) was reached on all but three statements according to the pre-defined criteria. The three statements without consensus were: 1) ERT should always be started in males, even if asymptomatic; 2) it is essential to assess Cystatin C at diagnosis; and 3 ) it is essential to assess Cystatin $C$ at follow-up.

\subsection{Diagnosis of $A F D$}

There was a negative consensus on four of five statements relating to diagnosis. None of the following were regarded as essential for diagnosis: the presence of angiokeratoma, cardiac involvement, renal alteration visible by abdominal ultrasound, and target-organ biopsy showing Gb3 deposits in men.

Regarding the requirement for detecting Gb3 deposits on targetorgan biopsy for diagnosis in women, negative consensus was obtained. When the phrases "when they present with signs and symptoms of disease" and "when the molecular analysis is negative" were added following discussion, positive consensus was then achieved. In addition, during discussion it also emerged that there was an agreement on detecting Gb3 in plasma and urine as a less invasive investigation in asymptomatic AFD patients.

\subsection{Starting ERT in patients with AFD}

Positive consensus was obtained on the initiation of ERT in all symptomatic women and in men with persistent signs and symptoms with or without organ damage (a new statement added after discussion). Statements regarding treating all patients with ERT from diagnosis, and use of ERT only if there is organ damage, both achieved negative consensus [one after revision], and the statement on treating all males even if asymptomatic, did not achieve consensus even after discussion.

\subsection{Management of ERT and adverse reactions in AFD}

Consensus was achieved on all statements, two on the second vote. Participants agreed that vital signs - heart rate, blood pressure and body temperature, as well as respiratory rate and oxygen saturation $\left(\mathrm{SATO}_{2}\right)$ - should be measured before and after ERT infusion. Positive consensus was increased after discussion with the immunologist to highlight the importance of using an integral $0.2 \mu \mathrm{L}$ IV filter with the agalsidase infusion line to eliminate aggregates, known to be a potential cause of infusion reactions.

In the event of a mild-to moderate adverse reaction, participants agreed that the infusion should be discontinued and restarted at a slower rate once the symptoms had regressed. However, with severe adverse reactions all participants agreed that the infusion should be stopped immediately and not restarted in the same day. In the case of adverse reactions the protocol for pre-medication should be the same as for other protein preparations. 
The need for determination of anti-agalsidase antibodies before starting treatment and in the case of an infusion reaction also achieved positive consensus. This was not surprising since the phenomenon of the development of neutralising antibodies to agalsidase and its adverse effect on efficacy is well known [15].

\subsection{Follow-up in AFD}

Ten statements on assessment to be performed at baseline and follow-up achieved positive consensus: Holter electrocardiography (ECG), cardiac magnetic resonance imaging (CMRI), microalbuminuria assessment, abdominal ultrasound and brain MRI. Two statements regarding the use of Cystatin $\mathrm{C}$ assessment at baseline and follow-up did not achieve consensus even after discussion.

\subsection{Management of pre-medication}

A section on pre-medication was added and discussed regarding agalsidase alfa only, whose prescribing information sheet does not include suggestions on premedication [16]. Conversely, the prescribing information sheet for agalsidase beta reports the need of premedication with antipyretics [17]. Participants disagreed that pre-medication should always be given before agalsidase alfa infusion. Positive consensus was achieved on the other two statements: pre-medication should only be given to patients with previous infusion reaction.

\section{Discussion}

In summary, all but four statements achieved consensus in the survey. After discussion of the contentious areas, lack of consensus remained for three statements relating to starting ERT in men regardless of symptoms, and assessment of Cystatin C at diagnosis or follow-up. There was negative consensus on four of five statements and positive consensus on one statement relating to diagnosis. Positive consensus was obtained on the initiation of ERT in all symptomatic women and in men with persistent signs with or without organ damage and symptoms and negative consensus was achieved for statements regarding treating all patients with ERT on diagnosis, and use of ERT only if there is organ damage. Regarding management of ERT and adverse reactions in AFD, consensus was achieved on all statements. In follow-up of patients with AFD, 10 statements on assessments to be performed at baseline and follow-up achieved positive consensus. A section on pre-medication in patients treated with agalsidase alfa was added and discussed.

The following provides a brief description of the discussions held on the contentious issues.

\subsection{Diagnosis}

All participants agreed after discussion that angiokeratoma is not present in all patients, especially at diagnosis. The presence of angiokeratoma correlates with age, rarely occurring during the first 10 years of age even in males with the classical form of the disease. Its presence is dependent on the form of AFD and correlates with severity $[18,19]$.

Although most participants thought that an abdominal ultrasound could be useful in patients with suspected renal involvement, from the clinical point of view, an abdominal ultrasound cannot lead to a differential diagnosis but does provide some additional renal information beyond proteinuria. It should be performed in all patients with suspected nephropathy regardless of its nature. Participants did not consider renal alteration at abdominal ultrasound essential for diagnosis. Cardiac involvement is common, but not considered essential to reach diagnosis. Data from the literature show that not all patients have a cardiac alteration on echography $[20,21]$.
In symptomatic women with negative molecular analysis, the use of a target-organ biopsy to show $\mathrm{Gb} 3$ deposits could be indispensable. In patients with positive molecular analysis, but negative signs and symptoms of illness, the less invasive investigation - detection of Gb3 in plasma and urine - can be used.

\subsection{Starting ERT}

No consensus was achieved for the statement "ERT should be started always in males, even if asymptomatic"; there are very mild mutations which do not cause any signs and symptoms, not even in males until they are elderly. Following discussion, the statement regarding starting ERT only if the parameters suggesting organ damage are altered, was rephrased as follows "ERT should be started only when the disease involves the heart, kidney and brain". The misleading part of the sentence was 'organ function parameters' since organ function can involve not only the heart and the kidney, but also hearing impairment, gastrointestinal disorders, quality of life, etc. for which there are no numerically measurable parameters. In this revised format, the statement achieved negative consensus. However, in view of this discussion, another statement "ERT should be started when the patient has persistent signs and symptoms with or without organ damage" was added and achieved positive consensus. The group favoured starting ERT at any age in diagnosed individuals (male or women) who show at least some persistent signs or symptoms of the disease after a complete multidisciplinary evaluation. There was no agreement on starting ERT in a completely asymptomatic patient only on the basis of mutation or family history.

\subsection{Management of ERT and adverse reactions}

In clinical practice, in addition to standard assessment of BP, HR, and body temperature, most clinicians measured respiratory rate and oxygen saturation even in patients with normal respiratory function.

There was some discussion regarding the $79 \%$ agreement on the statement about the use of an infusion line with integral $0.2 \mu \mathrm{L}$ filter for the administration of agalsidase to reduce particulate matter and microaggregates. The immunologists considered this to be of vital importance and were surprised that $21 \%$ of the clinicians did not agree with this statement, given that most infusion reactions are not related to antibody-mediated reactions, but are related predominantly to the presence of aggregates in solution [22]. Infusion procedures should be standardized for all protein derivatives in order to reduce the risk of adverse events which may be related to the presence of aggregates and complexes [22]. In addition to these clinical considerations, both ERT product summaries of product characteristics recommend the use of a filter [16,17]; therefore, there could be medical-legal issues if it is not used. After discussion, $100 \%$ positive consensus was achieved on the second vote.

For one statement, "I think that antibodies should be determined before starting the treatment", no consensus was achieved at the first vote. The importance of setting a baseline value, by which subsequent changes can be compared, was emphasized. From a practical point of view, serum can be taken and stored safely, as antibodies are stable over time, and then analysed if an adverse reaction occurs. After discussion, 95\% positive consensus was achieved. If adverse reactions occur, premedication is recommended following the use of a standard protocol for other protein preparations.

\subsection{Pre-medication}

Additional discussion, limited to agalsidase alfa, on the specific use of pre-medication, led to the addition of three statements relating to premedication, and positive consensus was achieved on the use of premedication only in patients with previous infusion reactions. 


\subsection{Follow-up}

Assessments related to cardiac involvement are particularly useful as abnormalities can be detected at an early stage before hypertrophy and fibrosis have developed [23]. Holter ECG monitoring is easy to do and it is particularly important in adolescents because clinical trials show that heart rate variability is altered in the early stage of disease before onset of hypertrophic cardiomyopathy [24].

cMRI is also a useful tool for assessing early cardiac involvement at diagnosis because it provides additional information to the Holter ECG, such as assessment of cardiac mass and detection of left ventricular hypertrophy [25]. It can detect the patterns of late gadolinium enhancement specific to AFD $[25,26]$ and has the advantage of being noninvasive. cMRI is equally important at follow-up and is especially useful for detecting fibrosis [27]. It provides information about disease progression and response to ERT, as patients with severe late enhancement do not respond to ERT $[28,29]$. The approach for using cMRI for diagnosis and follow-up is different in men and women due to gender differences in the patterns of cardiomyopathy seen [30]. A clinically important difference (progression or regression) can be detected by cMRI in as little as one year and therefore cMRI is helpful, but not essential. Unfortunately, the administration of gadolinium is contraindicated in patients with advanced renal insufficiency, so although very useful at follow-up, it is not always possible in patients with advanced AFD.

Regarding the assessment of microalbuminuria to detect nephropathy, in children and in all patients who do not provide appropriate 24-hour urine samples, an accepted method is to take the mean of microalbuminuria values from three consecutive morning urine samples - also measuring creatinine at the same time in order to assess the albumin-creatinine ratio; this method is used in patients with other diseases such as diabetes [31].

The use of brain MRI was discussed at length. It is considered to be useful at follow-up for monitoring of CNS damage, but not essential. A parenchymal MRI at follow-up is useful to determine if and when new ischemic lesions appear in order to associate them with clinical signs which may appear later on.

\subsection{Lack of consensus}

There was no consensus on three statements even after the plenary discussion and second voting. The statement "Enzyme replacement therapy should always be started in males, even if asymptomatic" reflects the previously discussed fact that mutations in males can cause a mild phenotype, only developing signs or symptoms at advanced age.
Although there was no consensus on the statements concerning assessment using Cystatin C at diagnosis ("I think it is essential to assess Cystatin C at patient diagnosis") and follow-up ("I think it is essential to assess Cystatin $C$ at patient follow-up") due to practical difficulties (an expensive test performed in few laboratories). It was pointed out that it could be a more appropriate test than glomerular filtration rate or creatinine serum level for monitoring the effect of treatment on renal function over time; therefore, clinicians should evaluate Cystatin C every 6 or 12 months [32-35]. Even if Cystatin C is tested only in specific high-risk patients (i.e. transplant patients), being an expensive examination, the importance of Cystatin $\mathrm{C}$ was emphasized and clinicians were urged to consider its use in a Fabry laboratory panel.

\section{Conclusions}

The Delphi method was used to obtain consensus on best practice on a range of topics related to diagnosis and treatment of patients with AFD in Italy. The method is well known for its robustness in making highly valid and unbiased consensus findings. The clinical recommendations of the Delphi panel experts (Table 1) are summarized as follows:

1. AFD diagnosis should be suspected in individuals of both sexes who show suggestive symptoms of AFD independently of the presence of angiokeratoma, or renal or cardiac alterations at ultrasound examination. A biopsy of target organs to detect Gb3 deposits should be performed to reach diagnosis in symptomatic women with no identified pathologic mutation. In asymptomatic patients with confirmed molecular diagnosis, Gb3 may be tested in plasma and urine.

2. ERT must be started in all women and men with persistent signs and symptoms.

3. An infusion line with integral $0.2 \mu \mathrm{L}$ filter should always be used for the administration of ERT.

4. A pre-treatment blood sample should be taken and stored. In the case of an adverse reaction to ERT, it will be tested for anti-agalsidase antibodies, together with another blood sample taken at the time of the reaction.

5. Premedication is recommended for agalsidase alfa therapy if the patient has had previous reactions.

6. Follow-up of the AFD patient should include Holter monitoring, microalbuminuria, abdominal echography and cardiac/brain MRI.

The results of this Delphi panel provide welcome guidance to healthcare providers on best practice in the management of patients with AFD. The outcomes of this Delphi panel show that some aspects of diagnosis and overall management of AFD need to be improved and should be addressed further.

Table 1

Clinical recommendations.

\begin{tabular}{|c|c|}
\hline Diagnosis & $\begin{array}{l}\text { In women presenting with signs and symptoms of disease, and negative molecular analysis, a Gb3 biopsy is essential. } \\
\text { In other patients, those with positive molecular analysis and no signs and symptoms, a less invasive option of detecting } \\
\text { Gb3 in plasma and urine can be useful. }\end{array}$ \\
\hline Starting ERT & $\begin{array}{l}\text { Enzyme replacement therapy should be started when the patient has persistent signs and symptoms with or without } \\
\text { organ damage. }\end{array}$ \\
\hline $\begin{array}{l}\text { Management of ERT agalsidase infusion and } \\
\text { adverse reactions }\end{array}$ & $\begin{array}{l}\text { It is important to assess vital signs (BP, } \mathrm{HR} \text {, respiratory rate, } \mathrm{SATO}_{2} \text { ) and temperature before the administration of ERT. } \\
\text { Use of an infusion line with integral filter (for } 0.2 \mu \mathrm{L} \text { intravenous infusion) is recommended. } \\
\text { Collect and store blood sample before the first infusion is started, so that pre-treatment anti-agalsidase antibody levels } \\
\text { can be evaluated together with current levels in the event of an adverse reaction. } \\
\text { If adverse reactions occur the prophylactic/therapeutic approach is similar to the one used for other protein infusion. }\end{array}$ \\
\hline $\begin{array}{l}\text { Pre-medication } \\
\text { Follow-up }\end{array}$ & $\begin{array}{l}\text { Before agalsidase alfa infusion, pre-medication should be given to patients who have previously had an infusion reaction. } \\
\text { At baseline and 6/12 months' follow-up the following are essential: }\end{array}$ \\
\hline & $\begin{array}{l}\text { - Holter monitoring to detect changes in heart rate variability } \\
\text { - Microalbuminuria } \\
\text { - Abdominal echography } \\
\text { At } 12 \text { months, the following may be useful: } \\
\text { - Cardiac magnetic resonance imaging } \\
\text { - Brain magnetic resonance imaging } \\
\text { There was NO consensus on assessment using Cystatin C. }\end{array}$ \\
\hline
\end{tabular}


Appendix A. Summary of all statements and responses

\begin{tabular}{|c|c|c|}
\hline & Diagnosis of Fabry disease & Consensus \\
\hline & 1. In order to have a diagnostic suspect of Fabry disease & \\
\hline 1 & 1.1 I think that the presence of angiokeratoma is essential & Negative consensus (96\%) \\
\hline 2 & 1.2 I think that abdominal echography is essential for the differential diagnosis with other renal diseases & Negative consensus (94\%) \\
\hline 3 & 1.3 I think it is essential to have a "renal" alteration at echocardiography & Negative consensus (90\%) \\
\hline 4 & 1.4 I think it is essential to have a target biopsy (i.e. skin, kidney) to show GB-3 deposits (in males) & Negative consensus \\
\hline 5 & $\begin{array}{l}1.5 \text { I think it is essential to have a target biopsy (i.e. skin, kidney) to show GB-3 deposits, (in women) "when they present } \\
\text { subsequent signs and symptoms of disease and no pathologic mutation is identified and the molecular analysis is negative" } \\
\text { When should the enzyme replacement therapy be started? }\end{array}$ & Negative consensus (91\%) \\
\hline 6 & 2.1 Always in patients of both sexes at diagnosis & Negative consensus \\
\hline 7 & $2.2^{*}$ Always in males, even if asymptomatic & No consensus \\
\hline 8 & 2.3. Always in symptomatic females & Positive consensus \\
\hline \multirow[t]{5}{*}{9} & 2.4 Only if the parameters suggesting organ damage are altered & Negative consensus (73\%) \\
\hline & 2.4rev Following discussion, the last question was rephrased as follows "Only when the disease involves heart, kidney and brain" & Negative consensus \\
\hline & 2.5 (added following discussion) When the patient has persistent signs and symptoms with or without organ damage & Positive consensus \\
\hline & Management of ERT adverse reactions in Fabry patient & \\
\hline & 1. I always measure $\mathrm{BP}, \mathrm{HR}$, respiratory rate, $\mathrm{SATO}_{2}$ and temperature & \\
\hline 10 & 1.1 Before the administration of ERT & Positive consensus (92\%) \\
\hline \multirow[t]{2}{*}{11} & 1.2 After the administration of ERT & Positive consensus (89\%) \\
\hline & 2. For the administration of agalsidase alfa & \\
\hline \multirow[t]{4}{*}{12} & 2.1 I should use an infusion line with integral filter (for $0.2 \mu \mathrm{l}$ IV infusion) (first vote) & Positive consensus (79\%) \\
\hline & 2.1 (after discussion with immunologists) I should use an infusion line with integral filter (for $0.2 \mu \mathrm{l}$ IV infusion) & Positive consensus (100\%) \\
\hline & Management of adverse reactions & \\
\hline & 3.1 In case of mild to moderate reactions to agalsidase alfa infusion & \\
\hline 13 & 3.1.1 I discontinue the infusion until regression of symptoms and then I start again at the same infusion rate & Negative consensus (84\%) \\
\hline 14 & 3.1.2 I discontinue the infusion until regression of symptoms and then I start again at a slower infusion rate & Positive consensus (92\%) \\
\hline 15 & 3.1.3 I reduce the rate without discontinuing the infusion & Negative consensus (94\%) \\
\hline \multirow[t]{2}{*}{16} & 3.1.4 I discontinue the infusion & Negative consensus (90\%) \\
\hline & 3.2 In case of severe adverse reactions to agalsidase alfa infusion & \\
\hline 17 & 3.2.1 I discontinue the infusion until regression of symptoms and then I start again at the same infusion rate & Negative consensus (97\%) \\
\hline 18 & 3.2.2 I discontinue the infusion until regression of symptoms and then I start again at a slower infusion rate & Negative consensus (87\%) \\
\hline 19 & 3.2.3 I reduce the rate without discontinuing the infusion & Negative consensus (98\%) \\
\hline \multirow[t]{2}{*}{20} & 3.2.4 I discontinue the infusion & Positive consensus (100\%) \\
\hline & Management of pre-medication in the treatment with agalsidase alfa & \\
\hline 21 & 4.1 I think that anti-ERT antibodies should be determined & \\
\hline 22 & 4.1.1* Before starting the treatment ( 2 nd vote [ 1 st vote no consensus $52 \%]$ ) & Positive consensus (95\%) \\
\hline \multirow[t]{3}{*}{23} & 4.1.2 In case of adverse reactions occurring during the infusion & Positive consensus (86\%) \\
\hline & Additional questions (added after final discussion) on management of pre-medication) & \\
\hline & 4.2 Before agalsidase alfa infusion & \\
\hline 1 & 4.2.1 Pre-medication should always be done? & Negative consensus \\
\hline 2 & 4.2.2 Only in patients who previously had an infusion reaction. & Positive consensus \\
\hline \multirow[t]{3}{*}{3} & 4.2.3 In case of adverse reaction, for the pre-medication I follow the same protocol that I follow for the other protein preparations. & Positive consensus \\
\hline & Follow-up in Fabry patients & \\
\hline & 1.1 I think it is essential to perform a Holter monitoring to detect changes in heart rate variability & \\
\hline 24 & 1.1.1 At diagnosis & Positive consensus (83\%) \\
\hline \multirow[t]{2}{*}{25} & 1.1.2 At follow-up & Positive consensus (95\%) \\
\hline & 1.2 I think it is indispensable to perform a cardiac magnetic resonance & \\
\hline 26 & 1.2.1 At diagnosis & Positive consensus (96\%) \\
\hline \multirow[t]{2}{*}{27} & 1.2.2 At follow-up & Positive consensus (92\%) \\
\hline & 1.3 I think it is essential to assess microalbuminuria & \\
\hline 28 & 1.3.1 At patient diagnosis & Positive consensus (100\%) \\
\hline \multirow[t]{2}{*}{29} & 1.3.2 At patient follow-up & Positive consensus (100\%) \\
\hline & 1.4 I think it is essential to assess cystatin C & \\
\hline 30 & 1.4.1* At patient diagnosis & No consensus \\
\hline \multirow[t]{2}{*}{31} & 1.4.2* At patient follow-up & No consensus \\
\hline & $1.5 \mathrm{I}$ think it is essential to perform an abdominal echography & \\
\hline 32 & 1.5.1 At patient diagnosis & Positive consensus (92\%) \\
\hline \multirow[t]{3}{*}{33} & 1.5.2 At patient follow-up & Positive consensus (74\%) \\
\hline & Neurological follow-up & \\
\hline & 1.6 I think it is essential to perform a brain magnetic resonance & \\
\hline 34 & 1.6.1 At patient diagnosis & Positive consensus (100\%) \\
\hline 35 & 1.6.2 At patient follow-up & Positive consensus (93\%) \\
\hline
\end{tabular}

Consensus is $100 \%$ unless stated otherwise.

* No consensus achieved in the pre-meeting survey (1st vote).

\section{Learning points}

- AFD should be suspected in individuals who show symptoms of disease regardless of the presence/absence of angiokeratoma, or cardiac/renal alterations at ultrasound examination.

- In symptomatic women with negative molecular analysis, a biopsy to detect Gb3 is required for diagnosis. In patients with positive molecular analysis, but negative signs and symptoms of illness, plasma and urine Gb3 can be assessed.

- ERT must be started in all patients with persistent signs and symptoms.

- An infusion line with integral $0.2 \mu \mathrm{L}$ filter should be used for the administration of ERT.

- A pre-treatment blood sample should be taken and stored. In the case of an adverse reaction to ERT, it will be tested for anti-agalsidase 
antibodies, together with another sample taken at the time of the reaction.

- Premedication is recommended for agalsidase alfa therapy if the patient has had previous reactions.

- Holter cardiac monitoring, microalbuminuria and abdominal ultrasound should be performed at 6- and 12-month follow-up, and cardiac and brain MRI can be useful at 12-month follow-up.

\section{Conflict of interests}

Daniela Concolino and Rossella Parini have received speaking fees from Shire, Genzyme SOBI and Bio Marin. Emilia Degennaro received Delphi panel organization fees from Shire HGT. The members of the Fabry Delphi working group have no conflicts of interest to declare.

\section{Acknowledgements}

The consensus panel was financially supported by Shire. We thank Health Publishing \& Services Srl who provided medical writing services on behalf of Shire. This service and the consensus panel were supported by a grant from Shire.

\section{Role of the funding source}

Shire had no involvement in the Delphi panel or in the collection, analysis and interpretation of data; in the writing of the report; and in the decision to submit the article for publication.

Daniela Concolino and Rossella Parini performed the analysis of survey responses, moderation of plenary discussions, review and approval of draft manuscript. Emilia Degennaro performed the analysis of survey responses and organised the Delphi panel. Members of the Delphi working group participated in the consensus meeting.

\section{References}

[1] Lidove O, West ML, Pintos-Morell G, Reisin R, Nicholls K, Figuera LE, et al. Effects of enzyme replacement therapy in Fabry disease-a comprehensive review of the medical literature. Genet Med 2010;12:668-79.

[2] Zarate YA, Hopkin RJ. Fabry's disease. Lancet 2008;372:1427-35.

[3] Parini R, Feriozzi S. Females and children with Anderson-Fabry disease: diagnosis, monitoring, benefits of enzyme replacement therapy (ERT) and considerations on timing of starting ERT. Expert Opin Orphan Drug 2013;1:315-30.

[4] Sestito S, Ceravolo F, Concolino D. Anderson-Fabry disease in children. Curr Pharm Des 2013:19:6037-45.

[5] Schiffmann R, Warnock DG, Banikazemi M, Bultas J, Linthorst GE, Packman S, et al. Fabry disease: progression of nephropathy, and prevalence of cardiac and cerebrovascular events before enzyme replacement therapy. Nephrol Dial Transplant 2009;24:2102-11.

[6] Warnock DG, Ortiz A, Mauer M, Linthorst GE, Oliveira JP, Serra AL, et al. Renal outcomes of agalsidase beta treatment for Fabry disease: role of proteinuria and timing of treatment initiation. Nephrol Dial Transplant 2012;27:1042-9.

[7] Keating GM. Agalsidase alfa: a review of its use in the management of Fabry disease. BioDrugs 2012;26:335-54.

[8] Rozenfeld P, Neumann PM. Treatment of Fabry disease: current and emerging strategies. Curr Pharm Biotechnol 2011;12:916-22.

[9] Ramaswami U. Update on role of agalsidase alfa in management of Fabry disease. Drug Des Devel Ther 2011;5:155-73.

[10] Watt T, Burlina AP, Cazzorla C, Schonfeld D, Banikazemi M, Hopkin RJ, et al. Agalsidase beta treatment is associated with improved quality of life in patients with Fabry disease: findings from the Fabry Registry. Genet Med 2010;12:703-12.
[11] Schiffmann R. Agalsidase treatment for Fabry disease: uses and rivalries. Genet Med 2010;12:684-5

[12] Motabar O, Sidransky E, Goldin E, Zheng W. Fabry disease - current treatment and new drug development. Curr Chem Genomics 2010;4:50-6.

[13] Mehta A, Beck M, Eyskens F, Feliciani C, Kantola I, Ramaswami U, et al. Fabry disease: a review of current management strategies. QJM 2010;103:641-59.

[14] Dalkey N, Brown B, Cochran S. The Delphi method, III: use of self ratings to improve group estimates. Santa Monica, CA: Rand Corporation; 1969.

[15] Linthorst GE, Hollak CE, Donker-Koopman WE, Strijland A, Aerts JM. Enzyme therapy for Fabry disease: neutralizing antibodies toward agalsidase alpha and beta. Kidney Int 2004;66:1589-95.

[16] Shire Human Genetic Therapies AB. Replagal (agalsidase alfa) $1 \mathrm{mg} / \mathrm{ml}$ concentrate for solution for infusion: summary of product characteristics; 2013 [Danderyd, Sweden].

[17] Genzyme Corporation. Fabrazyme (agalsidase beta) injection, powder, lyophilized for solution for intravenous use; 2010.

[18] Orteu CH, Jansen T, Lidove O, Jaussaud R, Hughes DA, Pintos-Morell G, et al. Fabry disease and the skin: data from FOS, the Fabry outcome survey. Br J Dermatol 2007; 157:331-7.

[19] Orteu CH, Larroque S, Mehta A, Gal A. Angiokeratoma in Fabry disease: significant correlation between genotype and phenotype. J Inher Metab Dis 2012, 35(Suppl 1):S94.

[20] Zamorano J, Serra V, Perez de Isla L, Feltes G, Calli A, Barbado FJ, et al. Usefulness of tissue Doppler on early detection of cardiac disease in Fabry patients and potential role of enzyme replacement therapy (ERT) for avoiding progression of disease. Eur J Echocardiogr 2011;12:671-7.

[21] Linhart A, Palecek T, Bultas J, Ferguson JJ, Hrudova J, Karetova D, et al. New insights in cardiac structural changes in patients with Fabry's disease. Am Heart J 2000 139:1101-8.

[22] Royal College of Nursing. Infusion equipment. Standard for infusion therapy: the RCN IV therapy forumThird ed. ; 2010.

[23] Koeppe S, Neubauer H, Breunig F, Weidemann F, Wanner C, Sandstede J, et al. MRbased analysis of regional cardiac function in relation to cellular integrity in Fabry disease. Int J Cardiol 2012;160:53-8.

[24] Kampmann C, Wiethoff CM, Whybra C, Baehner FA, Mengel E, Beck M. Cardiac manifestations of Anderson-Fabry disease in children and adolescents. Acta Paediatr 2008;97:463-9.

[25] Sado DM, White SK, Piechnik SK, Banypersad SM, Treibel T, Captur G, et al. Identification and assessment of Anderson-Fabry disease by cardiovascular magnetic resonance non-contrast myocardial T1 mapping. Circ Cardiovasc Imaging 2013;6:392-8.

[26] De Cobelli F, Esposito A, Belloni E, Pieroni M, Perseghin G, Chimenti C, et al. Delayedenhanced cardiac MRI for differentiation of Fabry's disease from symmetric hypertrophic cardiomyopathy. AJR Am J Roentgenol 2009;192:W97-W102.

[27] Weidemann F, Breunig F, Beer M, Sandstede J, Stork S, Voelker W, et al. The variation of morphological and functional cardiac manifestation in Fabry disease: potential implications for the time course of the disease. Eur Heart J 2005;26:1221-7.

[28] Messalli G, Imbriaco M, Avitabile G, Russo R, Iodice D, Spinelli L, et al. Role of cardiac MRI in evaluating patients with Anderson-Fabry disease: assessing cardiac effects of long-term enzyme replacement therapy. Radiol Med 2012;117:19-28.

[29] Beer M, Weidemann F, Breunig F, Knoll A, Koeppe S, Machann W, et al. Impact of enzyme replacement therapy on cardiac morphology and function and late enhancement in Fabry's cardiomyopathy. Am J Cardiol 2006;97:1515-8.

[30] Niemann M, Herrmann S, Hu K, Breunig F, Strotmann J, Beer M, et al. Differences in Fabry cardiomyopathy between female and male patients: consequences for diagnostic assessment. JACC Cardiovasc Imaging 2011;4:592-601.

[31] KDOQI. KDOQI clinical practice guideline for diabetes and CKD: 2012 update. Am J Kidney Dis 2012:60:850-86.

[32] Feriozzi S, Germain DP, Di Vito R, Legrand A, Ricci R, Barbey F. Cystatin C as a marker of early changes of renal function in Fabry nephropathy. J Nephrol 2007;20:437-43.

[33] Torralba-Cabeza MA, Olivera S, Hughes DA, Pastores GM, Mateo RN, Perez-Calvo JI. Cystatin C and NT-proBNP as prognostic biomarkers in Fabry disease. Mol Genet Metab 2011;104:301-7.

[34] Rombach SM, Baas MC, ten Berge IJ, Krediet RT, Bemelman FJ, Hollak CE. The value of estimated GFR in comparison to measured GFR for the assessment of renal function in adult patients with Fabry disease. Nephrol Dial Transplant 2010;25:2549-56.

[35] Tondel C, Ramaswami U, Aakre KM, Wijburg F, Bouwman M, Svarstad E. Monitoring renal function in children with Fabry disease: comparisons of measured and creatinine-based estimated glomerular filtration rate. Nephrol Dial Transplant 2010;25:1507-13. 